\title{
The radio continuum of the extremely metal-poor blue compact dwarf galaxy I Zw 18
}

\author{
L. K. Hunt ${ }^{1}$, K. K. Dyer ${ }^{2}$, and T. X. Thuan ${ }^{3}$ \\ 1 INAF - Istituto di Radioastronomia/Sez. Firenze, Largo Enrico Fermi, 5, 50125 Firenze, Italy \\ e-mail: hunt@arcetri.astro.it \\ 2 Naval Research Lab, Code 7213 Washington, DC 20375-535, USA \\ e-mail: Kristy.Dyer@nrl .navy.mil \\ 3 Astronomy Department, University of Virginia, PO Box 3818, University Station, Charlottesville, VA 22903, USA \\ e-mail: txt@virginia.edu
}

Received 21 February 2005 / Accepted 10 March 2005

\begin{abstract}
We present 1.4, 4.8 and $8.4 \mathrm{GHz}$ Very Large Array observations of the lowest metallicity blue compact dwarf (BCD) galaxy known, IZw 18, with a heavy element abundance of $\sim 2 \%$ that of the Sun. The 1.4 and $4.8 \mathrm{GHz}$ images reveal a halo of mostly non-thermal extended emission, with asymmetric lobes extending laterally in the east-west direction. We interpret the radio halo as produced by a superbubble carved out in the interstellar medium (ISM) by supernovae, with a bipolar outflow oriented along the galaxy's rotation axis. The overall spectral index is -0.39 from $1.4 \mathrm{Ghz}$ to $4.8 \mathrm{Ghz}$ and -0.13 from 4.8 to 8.4 GHz. The radio luminosity of IZw 18 has a thermal to total emission fraction of 0.30 at $1.4 \mathrm{GHz}$. This fraction increases to 0.41 at $4.8 \mathrm{GHz}$ and to 0.47 at $8.4 \mathrm{GHz}$. The thermal radio luminosity gives a total of $1200 \mathrm{O} 7 \mathrm{~V}$ stars and a star formation rate of $0.1 M_{\odot} \mathrm{yr}^{-1}$. Unlike the BCD SBS 0335-052 which has a similar metallicity and forms stars with a high rate in regions which are dense and compact, IZw 18 makes stars at a smaller rate in complexes which are diffuse and extended. Star formation in BCDs thus appears to occur in a bimodal fashion, independently of the metallicity of the interstellar medium.
\end{abstract}

Key words. galaxies: dwarf - galaxies: starburst - galaxies: star clusters - radio continuum: galaxies radio continuum: ISM - ISM: supernova remnants

\section{Introduction}

How and when galaxies formed is a fundamental problem of modern astrophysics. To date however the quest for truly young galaxies in the process of forming at redshifts $>5-6$ is still unsuccessful. The high-redshift objects discovered so far are typically enriched in heavy elements, implying that the starformation events which formed them took place in an earlier yet unobserved epoch. Instead of looking for young systems at high redshifts, an alternative approach is to study star formation in environments which are similar to primordial ones, in that they are unevolved chemically. Chemically unenriched interstellar media in the local universe, such as those in metalpoor blue compact dwarf galaxies (BCDs), approximate the best those which hosted primordial starbursts; but because they are nearby, they are easier to study than the high-redshift Ly $\alpha$ absorption systems. Therefore, low-metallicity BCDs may be useful "templates" for studying how the first stars and galaxies formed.

Because of its extremely low metal abundance, IZw 18 is arguably one of the best nearby BCDs with which to study primordial-like starbursts. First discovered by Zwicky (1966), IZw 18 was originally described as a double system of compact galaxies, which are instead two massive star clusters, the brighter northwest (NW) and fainter southeast (SE) components, separated by $\sim 300 \mathrm{pc}$. The galaxy, together with a starforming region $\sim 1.3 \mathrm{kpc}$ to the NW (component C), is embedded within a massive HI cloud (van Zee et al. 1998).

I Zw 18 has the lowest metal abundance $\left(\sim 1 / 50 Z_{\odot}\right.$ Izotov et al. 2001, and references therein) of known star-forming systems in the local universe. Very metal-deficient objects with $Z \lesssim Z_{\odot} / 20$ are rare (e.g., Kunth \& Östlin 2000). Consequently IZw 18 has been studied extensively over the past several decades since the first determination of its heavy-element abundance (Sargent \& Searle 1970). One of the main reasons for such scrutiny is the idea, first proposed by Searle \& Sargent (1972), that low-metallicity dwarf galaxies such as IZw 18 are young, forming the bulk of their stars at a relatively recent epoch. For IZw 18, this seems to be true. While most BCDs are surrounded by low-surface brightness envelopes of evolved several Gyr old stars formed in previous bursts of star formation (Loose \& Thuan 1986), the colors of the emission in the outer regions of IZw 18 correspond to ionized gas, rather than to an old stellar population (Papaderos et al. 2002; Hunt et al. 2003). Moreover, deep images obtained with the ACS on the 
Hubble Space Telescope (HST) show that there are no red giant stars in the color-magnitude diagram (Izotov \& Thuan 2004). Hence, IZw 18 seems to be a genuinely young galaxy, with stellar populations no older than $\sim 500 \mathrm{Myr}$. As such it is one of the best nearby "laboratories" where we can investigate chemically unenriched star formation, and its effect on the interstellar medium (ISM).

Radio continuum emission is an important diagnostic of star-formation processes because of its direct link to the starburst component through both thermal and non-thermal emission. Because radio wavelengths are unaffected by dust and thus do not require uncertain extinction corrections, the radio region has become a favored one for measuring the starformation rate (SFR) as a function of lookback time (e.g., Haarsma et al. 2000). Scaling laws derived locally (Condon 1992) are used for this, but it is not clear how well such relations hold for extremely high redshift objects. They do not hold for local young starbursts (e.g., Roussel et al. 2003), nor do they hold for most local dwarf galaxies (e.g., Beck et al. 2000, 2002; Hunt et al. 2005). Should these dominate in the early universe, the SFRs derived for high-redshift objects need to be reassessed.

In a previous paper (Hunt et al. 2004, hereafter Paper I), we have investigated the radio continuum of SBS 0335-052, another BCD with extremely low metallicity $\left(1 / 41 Z_{\odot}\right.$, Thuan et al. 1997). In SBS 0335-052, there is substantial free-free absorption at $1.4 \mathrm{GHz}$, together with a non-typical mix of thermal/non-thermal emission. Moreover, the thermal radio flux has a high emission measure, and spectral fits suggest that the source is compact, $\sim 19$ pc in diameter, and dense, $n_{\mathrm{e}} \sim 2000 \mathrm{~cm}^{-3}$.

In this paper, we study the radio continuum of IZw 18 , with Very Large Array (VLA) images at 1.4, 4.8 and $8.4 \mathrm{GHz}$. The data reduction and analysis are described in Sect. 2. We describe the continuum morphology in Sect. 3, and the spectral fits in Sect. 4. Finally, in Sect. 5, we discuss IZw 18 in the context of star-formation diagnostics, and compare it with SBS 0335-052 and other low-metallicity dwarf galaxies. We adopt 14.0 Mpc (Izotov \& Thuan 2004) as the distance to IZw 18 . This distance is slightly larger than the one of 12.6 Mpc proposed before for IZw 18 (Östlin 2000) so that the absolute magnitudes of the asymptotic giant branch stars in I Zw 18 are comparable to those in other BCDs.

\section{VLA observations, reduction and analysis}

VLA $^{1}$ observations of IZw 18 from programs AK331 and AD440 were obtained from the NRAO archives ${ }^{2}$. The observations covered the frequencies $1.4 \mathrm{GHz}$ ( $L$ band), $4.8 \mathrm{GHz}$ ( $C$ band), and $8.4 \mathrm{GHz}$ ( $X$ band), and are reported in Table 1. The data were calibrated and imaged following standard procedures in the AIPS Cookbook. B1950 coordinates were precessed to $\mathrm{J} 2000$ using $U V F I X$. Since the flux calibrator

\footnotetext{
${ }^{1}$ The Very Large Array/National Radio Astronomy Observatory is a facility of the National Science Foundation operated under cooperative agreement by Associated Universities, Inc.

2 archive.nrao.edu
}

Table 1. VLA observations of IZw 18.

\begin{tabular}{ccccc}
\hline \hline Date & OBSID & Conf. & $\begin{array}{c}\text { Frequency } \\
{[\mathrm{GHz}]}\end{array}$ & $\begin{array}{c}\text { Time on source } \\
{[\mathrm{m}]}\end{array}$ \\
\hline 1 Aug. 93 & AK331 & $\mathrm{C}$ & 4.8 & 15 \\
22 Dec. 93 & AK331 & D & 8.4 & 18 \\
9 Jun. 94 & AK331 & B & 1.4 & 13 \\
20 Oct. 00 & AD440 & A & 1.4 & 13 \\
20 Oct. 00 & AD440 & A & 4.8 & 13 \\
2 Feb. 01 & AD440 & AB & 1.4 & 13 \\
\hline
\end{tabular}

Table 2. Images analyzed for I Zw 18.

\begin{tabular}{|c|c|c|c|}
\hline $\begin{array}{c}\text { Frequency } \\
{[\mathrm{GHz}]}\end{array}$ & Conf. & $\begin{array}{c}\text { Beam } \\
{[\text { Size, } \mathrm{PA}]}\end{array}$ & $\begin{array}{c}\text { rms noise } \\
{[\mu \mathrm{Jy}]}\end{array}$ \\
\hline \multicolumn{4}{|c|}{ Nondetections } \\
\hline 1.4 & A & $2^{\prime \prime} .2 \times 1^{\prime \prime} .3,89^{\circ}$ & 52 \\
\hline 4.8 & A & $0^{\prime \prime} 7 \times 0^{\prime \prime} .4,81^{\circ}$ & 54 \\
\hline \multicolumn{4}{|c|}{ "Best" images } \\
\hline 1.4 & $\mathrm{~A}, \mathrm{AB}, \mathrm{B}$ & $4^{\prime \prime} 6 \times 33^{\prime \prime} 7,-71^{\circ}$ & 56 \\
\hline 4.8 & $\mathrm{C}$ & $5^{\prime \prime} .2 \times 4^{\prime \prime} .4,12^{\circ}$ & 55 \\
\hline 8.4 & $\mathrm{D}$ & $10^{\prime} .5 \times 8^{\prime \prime} .8,-19.0^{\circ}$ & 40 \\
\hline \multicolumn{4}{|c|}{ Spectral index map } \\
\hline 1.4 & B & $5^{\prime \prime} .2 \times 5^{\prime \prime} .2$ & 70 \\
\hline 4.8 & $\mathrm{C}$ & $5^{\prime \prime} .2 \times 5^{\prime \prime} .2$ & 54 \\
\hline
\end{tabular}

(3C 286) was resolved in the A-configuration, following the AIPS Cookbook prescription, we used a restricted UV range $(90-180 \mathrm{k} \lambda)$ and reduced flux. Self-calibration failed to improve the signal to noise, therefore we present only standard calibration maps.

\subsection{Data analysis}

We created several images of IZw 18 at each frequency, for a series of scientific goals. These image parameters are listed in Table 2 and described briefly here. First, we produced images at the highest possible resolutions: $0.7 \times 0.4$ at $4.8 \mathrm{GHz}$ and 2 .' $2 \times 1$ 1.' 3 at $1.4 \mathrm{GHz}$. The source IZw 18 was not detected in these images - we discuss the significance of this nondetection in 3.1. Second, we made "best" images at each frequency: $\mathrm{A}+\mathrm{B}+\mathrm{AB}$ at $1.4 \mathrm{GHz}, \mathrm{C}$ at $4.8 \mathrm{GHz}$, and $\mathrm{D}$ at $8.4 \mathrm{GHz}$ ( since we lacked $\mathrm{B}$ configuration we chose not to add $\mathrm{A}+\mathrm{C}$ at $4.8 \mathrm{GHz}$ ); these images combine the best signalto-noise and the best angular resolution in the archive images. Because a change in the UV coverage can change both the total flux measured and the flux distribution in an image, we created UV-similar images for the spectral index images. We chose data from scaled arrays (B at $1.4 \mathrm{GHz}, \mathrm{C}$ at $4.8 \mathrm{GHz}$ ), used a UV taper to discard baselines, and CONVL to convert the images to identical 5". 2 resolutions. 

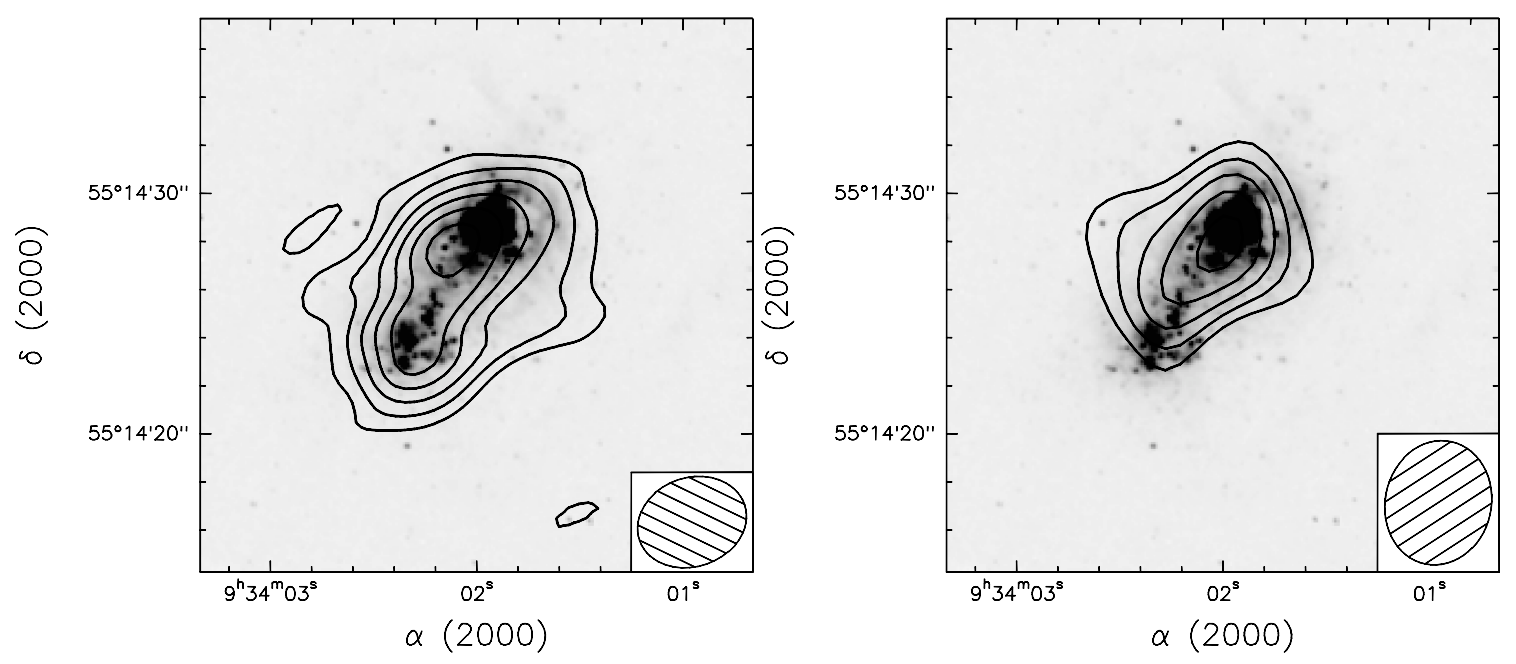

Fig. 1. Radio continuum contours of IZw 18 superimposed on the HST WFPC2/F555W image used in Hunt et al. (2003); the 1.4 GHz image is shown in the left panel, and the $4.8 \mathrm{GHz}$ image in the right panel. Radio contours in both panels go from $150 \mu \mathrm{Jy} \mathrm{beam}^{-1}(3 \sigma)$ to $5 \mathrm{mJy}$, in units of $\sigma\left(=50 \mu \mathrm{Jy}_{\text {beam }}{ }^{-1}\right)$. The beams are shown in the lower right corner of each panel.

\subsubsection{Optical-radio alignment}

We performed an astrometric calibration using stars from the US Naval Observatory Astrometric Catalog B1.0 (USNO-B1.0). There are five stars from this catalogue in the $1600 \times 1600$ WFPC 2 image of IZw 18 and they were used to derive the astrometric solution with imwcs in the WCSTools package $^{3}$. The solution has an rms uncertainty of 0 ' 37 , or roughly 4 WFPC2 (mosaic) pixels. The two highest-resolution best radio continuum maps are shown superimposed on the HST WFPC2/F555W image in Fig. 1. The optical continuum image is the same as the one used in Hunt et al. (2003).

\subsubsection{Spectral index map}

The spectral index (SI) map was created from UV-similar images with a $5^{\prime \prime} 2$ resolution, clipped to $4 \sigma$. Figure 2 shows the SI $\alpha_{21}^{6}$ map in gray scale, with the $1.4 \mathrm{GHz}$ continuum contours from Fig. 1 superimposed. Since the SE region is significantly fainter than the $\mathrm{NW}$ region at $4.8 \mathrm{GHz}$, it does not appear in the SI image.

\subsubsection{Total flux measurements}

Global fluxes were derived from the best images with polygonal and circular apertures using IRAF ${ }^{4}$. Background levels were determined in empty regions adjacent to the galaxy, then subtracted from the integrated flux. Table 3 gives the global fluxes and their uncertainties. The uncertainties are taken to be the standard deviations between the values in the circular and polygonal apertures. They are always larger than or comparable to the uncertainties due to the noise of the VLA image

\footnotetext{
${ }^{3}$ Available from http://tdc-www.harvard.edu/software/ wcstools

${ }^{4}$ IRAF is the Image Analysis and Reduction Facility made available to the astronomical community by the National Optical Astronomy Observatory, which is operated by AURA, Inc., under contract with the US National Science Foundation.
}

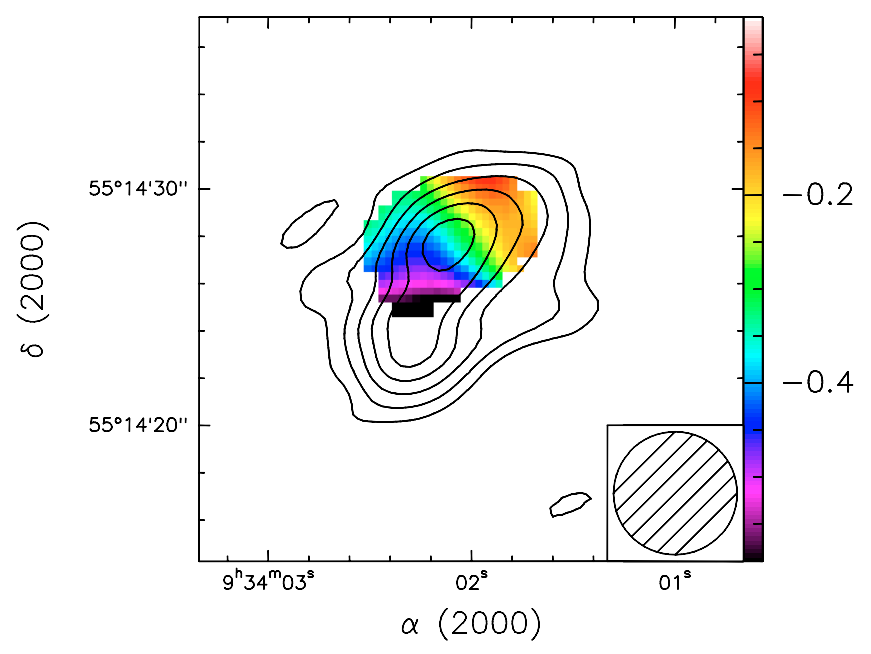

Fig. 2. The $4 \sigma$ spectral index map (between 1.4 and $4.8 \mathrm{GHz}$ ), with $4.8 \mathrm{GHz}$ contours (see Fig. 1) superimposed. The 5'.2 beam of the $\mathrm{UV}$-similar images is shown in the lower right corner.

combined with flux calibration uncertainties. These results agree satisfactorily with measurements using TVSTAT and IRING in AIPS.

\section{Results}

Our best images are sensitive to extended emission, but have limited spatial resolution. In what follows, we will therefore emphasize the large-scale morphology of our radio images and what it tells us about star formation in IZw 18.

\subsection{Limits to the size of the radio sources in IZw 18}

The most striking feature in these radio data is the lack of strong point sources in our A-configuration data at 1.4 and $5 \mathrm{GHz}$. Inspection of Fig. 1 shows that the radio emission is extended with no evidence of unresolved structure to the noise 

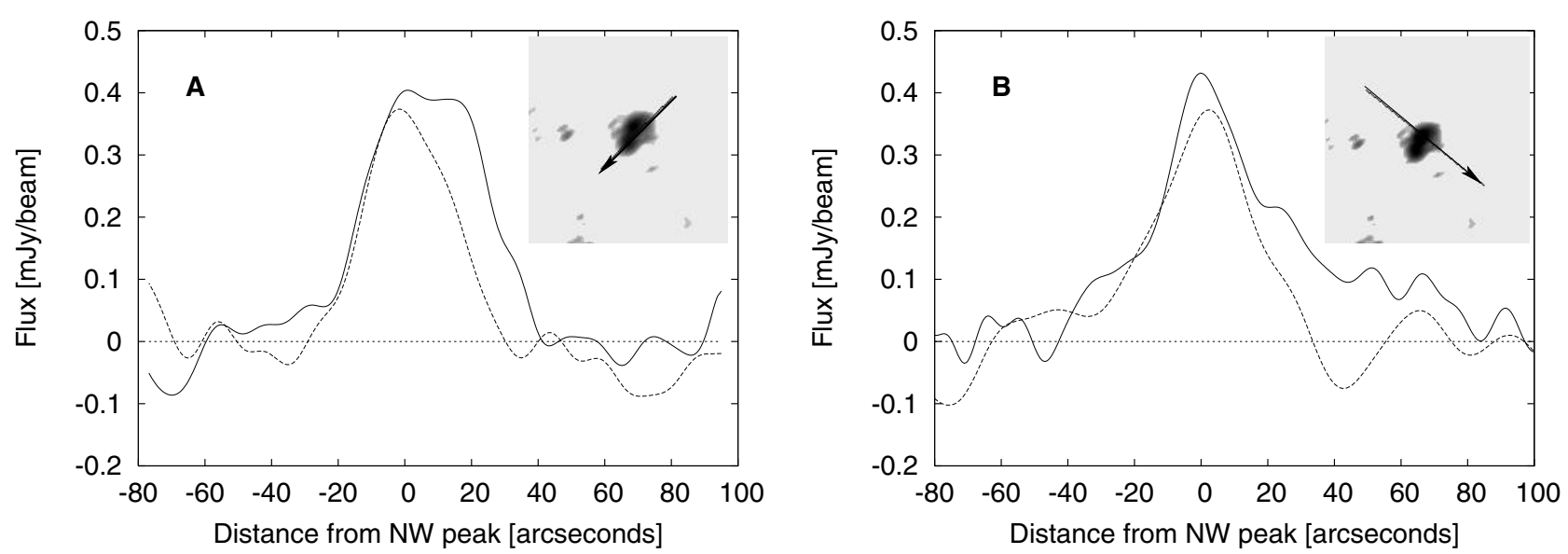

Fig. 3. Slices through the best 1.4 (solid line) and $4.8 \mathrm{GHz}$ (dashed line) images at a) PA $135^{\circ}$ (left panel) and b) $225^{\circ}$ (right). The cuts are centered on the $1.4 \mathrm{GHz}$ brightness peak. Distances increase in the SE direction. The illustrations in the upper right corners of both panels show the orientation of the cuts.

Table 3. Flux measurements of IZw 18.

\begin{tabular}{lrrrl}
\hline \hline $\begin{array}{c}\text { Frequency } \\
{[\mathrm{MHz}]}\end{array}$ & $\begin{array}{r}\text { Bandwidth } \\
{[\mathrm{MHz}]}\end{array}$ & Beam & Flux & Telescope \\
\hline 1416.8 & 1.25 & $27^{\prime \prime} \times 33^{\prime \prime}$ & $2.5 \pm 0.9$ & Westerbork $^{a}$ \\
$1464.9 / 1385.1$ & $2 \times 50$ & $11^{\prime \prime} \times 11^{\prime \prime}$ & $1.83 \pm 0.33$ & VLA $^{b}$ \\
4750 & 500 & $144^{\prime \prime} \times 144^{\prime \prime}$ & $1.6 \pm 0.2$ & Effelsberg $^{c}$ \\
$4885.1 / 4835.1$ & $2 \times 50$ & $11^{\prime \prime} \times 11^{\prime \prime}$ & $1.14 \pm 0.14 \mathrm{VLA}^{b}$ \\
$8414.9 / 8464.9$ & $2 \times 50$ & $11^{\prime \prime} \times 11^{\prime \prime}$ & $1.06 \pm 0.07$ & VLA $^{b}$ \\
10700 & 500 & $72^{\prime \prime} \times 72^{\prime \prime}$ & $2.1 \pm 0.6$ & Effelsberg $^{c}$ \\
\hline
\end{tabular}

${ }^{a}$ Lequeux \& Viallefond (1980).

${ }^{b}$ This work.

${ }^{c}$ Klein et al. (1983), Gioia et al. (1982).

levels of our images. Any point source would have to have an integrated flux $\lesssim 250 \mu \mathrm{Jy}$.

We have also investigated what we would observe in the A configuration at $1.4 \mathrm{GHz}$ if the source were slightly extended, but with the same total flux of $1.8 \mathrm{mJy}$, as detected in our image. As a test, we have added to our map an unresolved point source and three circular Gaussians with full width half maxima $(F W H M)$ of $4^{\prime \prime}, 5^{\prime \prime}$ and $6^{\prime \prime}$. We found that if the radio emission in I Zw 18 were a point source or slightly resolved (up to $4^{\prime \prime}$ ) we would have detected it in our A-configuration images. However, we would not have detected a $1.8 \mathrm{mJy}$ source larger than 5". At a distance of $14 \mathrm{Mpc}$, this translates into a lower limit of $\sim 340 \mathrm{pc}$.

We repeated the same experiment at $5 \mathrm{GHz}$ in the A configuration with a point source and Gaussians of 1'. 0,1 ,'5, and 2.'0 FHWM; all the sources had the same total flux, $1.1 \mathrm{mJy}$, as that detected in our $5 \mathrm{GHz}$ image. With our A-configuration image we would have detected the point source, and a Gaussian of $\lesssim 1^{\prime \prime}$. 5 , but if the source were larger than this $(\sim 135 \mathrm{pc})$, we would have missed it.

\subsection{Radio continuum morphology}

The morphology of the radio emission illustrated in Fig. 1 is rather peculiar. The $1.4 \mathrm{GHz}$ radio peak at (J2000) $\alpha=$ $9^{\mathrm{h}} 34^{\mathrm{m}} 02^{\mathrm{s}}, \delta=55^{\circ} 14^{\prime} 29^{\prime \prime}$ is centered just outside the NW cluster optical continuum peak, slightly to the SE. The $1.4 \mathrm{GHz}$ emission is extended in a halo-like structure, elongated along the axis connecting the NW-SE star clusters, with faint emission (at the $3 \sigma$ level) in lateral protuberances resembling "ears". The $4.8 \mathrm{GHz}$ image shows similar structure in the extended emission. The radio peaks at both frequencies lie outside the star clusters, and are slightly offset from one another: while the $1.4 \mathrm{GHz}$ emission peak is to the southeast of the $\mathrm{NW}$ cluster, the $4.8 \mathrm{GHz}$ peak is more to the south of it. I Zw 18 is unresolved by the $8.4 \mathrm{GHz} \mathrm{D}$ configuration with a resolution of 10 .' $5 \times 8$.' 8 . The $8.4 \mathrm{GHz}$ emission (not shown) peaks between the two NW and SE clusters.

The $4.8 \mathrm{GHz}$ image shows significantly less extension to the $\mathrm{SE}$ than the $1.4 \mathrm{GHz}$ emission. Figure 3 shows an intensity slice through $\mathrm{IZw} 18$ along the line connecting the NW and SE clusters with a position angle of $135^{\circ}$ (left panel), compared to the cut orthogonal to that NW-SE line with a position angle of $225^{\circ}$ (right panel). Toward the SE cluster, the $4.8 \mathrm{GHz}$ emission (dashed line) is clearly weaker than the $1.4 \mathrm{GHz}$ emission (solid line); however, perpendicular to the NW-SE axis, the extension is more similar at the two frequencies.

\subsection{A possible thermal source}

The structure of the SI map shown in Fig. 2 indicates that the spectral index steepens from the NW to the SE in IZw 18. In the northernmost parts of NW component, the spectral index is approximately thermal $\left(\alpha_{21}^{6} \sim-0.1--0.2\right)$, but toward the south, $\alpha \sim-0.5$. As mentioned before, the southern extension of IZw 18 is too faint at $4.8 \mathrm{GHz}$ to appear in the SI map.

The NW star cluster is associated with a region of thermal emission. However, the position of the flat spectral index does not correspond to the cluster optical peak, or to any radio brightness maximum. 

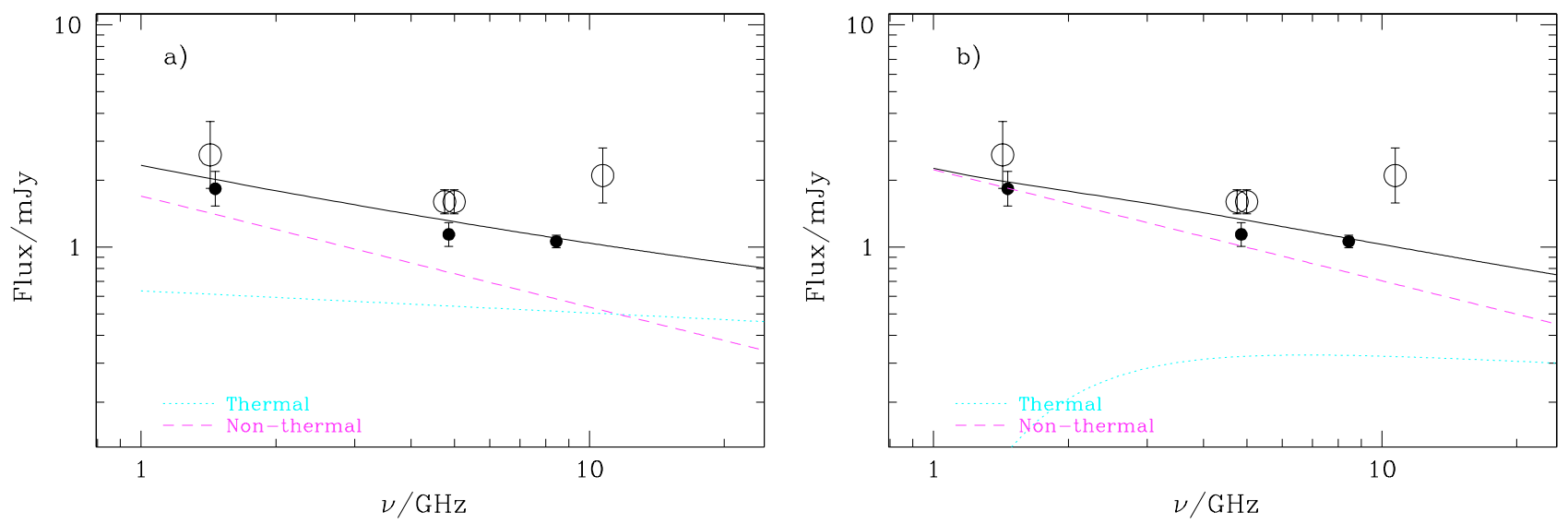

Fig. 4. Global radio continuum flux versus frequency $v$. The left panel a) shows the preferred best-fit solution, with $E M=7.9 \times 10^{3} \mathrm{pc} \mathrm{cm}^{-6}$, and thermal source size $9^{\prime \prime}(\sim 600 \mathrm{pc})$. The right panel b) shows the more extreme best-fit solution, with $E M=(1.9 \pm 1.9) \times 10^{7} \mathrm{pc} \mathrm{cm}^{-6}$, and source size $\sim 11 \mathrm{pc}$. The small filled circles are the results from this paper; the large open circles are single-dish observations from Klein et al. (1991). In each panel, a solid line shows the best-fit model, with the separate components given by a dotted line (thermal) and a dashed one (non-thermal). The spectral index for the non-thermal component $\alpha$ is -0.5 .

Another indication that there may be a thermal source to the NW is the asymmetry in the cuts (see Fig. 3). The emission is more compact at 4.8 than at $1.4 \mathrm{GHz}$, and more confined to the NW region.

\section{Modeling the global radio spectrum in IZw 18}

The global spectral index from 1.4 to $4.8 \mathrm{GHz}$ is $\alpha_{21}^{6}=-0.39$, suggesting a significant non-thermal contribution to the observed flux. This is a slightly steeper index than the mean $\alpha=-0.3$ of the BCD sample studied by Klein et al. (1984). The SI map in Fig. 2 indicates generally flatter indices because it traces mainly the NW portion of IZw 18 , the SE $4.8 \mathrm{GHz}$ emission being too faint. The spectral index steepens to the SE, making the global spectral index steeper. The global spectral index from 4.8 to $8.4 \mathrm{GHz}$ is thermal, $\alpha_{6}^{3.6}=-0.13$.

To better establish the relative contribution between thermal and non-thermal processes, we modeled the global flux using the formalism given by Paper I. Briefly, the global fluxes are fit by a thermal source extinguished by a screen of free-free absorption, together with a non-thermal source of known spectral index. The free parameters in the fit are the thermal emission measure $(E M)$, the thermal source size $\theta$, and the non-thermal flux $f_{v_{0}}^{\text {nt }}$ at frequency $v_{0}$. With only three frequencies, such a fit is underconstrained with our data alone. Hence, we used all of the available flux measurements, including single-dish values at 1.4, 4.8, and $10.7 \mathrm{GHz}$ collected in Klein et al. (1991); these are given in Table 3. We tried three values of $\alpha$ for the nonthermal source, including $\alpha=-0.4,-0.5-0.8$. The electron temperature was taken to be $19000 \mathrm{~K}$ (Izotov et al. 2001).

The results of the model fits are shown in Fig. 4. The left panel shows the most physically likely scenario in which the thermal source is $9^{\prime \prime}$ in diameter, and the $E M$ is $7.9 \pm 6.7 \times$ $10^{3} \mathrm{pc} \mathrm{cm}^{-6}$; the non-thermal source has a $5 \mathrm{GHz}$ flux of $0.76 \pm$ $0.38 \mathrm{mJy}$. The reduced $\chi_{v}^{2}$ of this fit is 2.5 . The right panel shows a less physically likely but equally significant result, also with $\chi_{v}^{2}=2.5$. Here, the thermal source $E M$ is $1.9 \pm 1.9 \times$ $10^{7}$, the source size $0.15^{\prime \prime}(10 \mathrm{pc})$, and the non-thermal flux $f_{v_{0}}^{\mathrm{nt}}=1.0 \pm 0.5 \mathrm{mJy}$. In this case, the $E M$ is more poorly determined, and the source size is unrealistically small. If we eliminate the highly discrepant $10 \mathrm{GHz}$ point from the dataset, the fit is significantly improved $\left(\chi_{v}^{2}=2.2\right)$, giving results similar to the first fit.

We therefore adopt the first fit on the complete dataset, which gives a thermal flux at $4.8 \mathrm{GHz}$ of $0.54 \mathrm{mJy}$, with a thermal to total flux ratio of $0.30,0.41$, and 0.47 at $1.4,4.8$, and $8.4 \mathrm{GHz}$, respectively. At these frequencies, the non-thermal flux is $1.43,0.77,0.59 \mathrm{mJy}$, respectively. The thermal source size is not well constrained in this fit, since with sizes anywhere from 9 to $30^{\prime \prime}$ in diameter, a similarly good fit is achieved (for a given thermal flux, the diameter squared and the $E M$ are inversely correlated). The quality of the fit worsens for sizes $<9^{\prime \prime}$, so we took $9^{\prime \prime}(\sim 600 \mathrm{pc}$ at a distance of $14 \mathrm{Mpc})$ as the best estimate. The low $E M$ given by this fit does not induce a noticeable turnover at low frequencies, in accordance with the observations.

The different best fits for the three values of the non-thermal spectral index $\alpha$ clearly show that $\alpha \lesssim-0.5$. Similar $\chi^{2}$ values were obtained for $\alpha=-0.4$ and -0.5 , but the fit is significantly degraded for $\alpha=-0.8$.

\subsection{Thermal and non-thermal emission}

From the spectral fit, we can better estimate the relative thermal/non-thermal contributions to the radio emission in IZw 18. The non-thermal fraction at $5 \mathrm{GHz}$ is 0.43 , with a non-thermal $5 \mathrm{GHz}$ flux of $0.52 \mathrm{mJy}$. The thermal emission at $5 \mathrm{GHz}, 0.68 \mathrm{mJy}$, can be compared to that expected from the observed optical recombination line flux (Hunter \& Thronson 1995; Martin 1996; Petrosian et al. 1997; Cannon et al. 2002). Using the formulations valid for electron temperatures greater than $10000 \mathrm{~K}$ (Paper I) and an electron temperature of $19000 \mathrm{~K}$ (Izotov et al. 2001), the $\mathrm{H} \alpha$ flux predicted from the free-free radio flux would be $4.6 \times 10^{-13} \mathrm{erg} \mathrm{cm}^{-2} \mathrm{~s}^{-1}$, consistent with the observations of Hunter \& Thronson $\left(1995, F_{\mathrm{H} \alpha}=3.9 \times 10^{-13} \mathrm{erg} \mathrm{cm}^{-2} \mathrm{~s}^{-1}\right.$ ), 
Martin $\left(1996, F_{\mathrm{H} \alpha}=5 \times 10^{-13} \mathrm{erg} \mathrm{cm}^{-2} \mathrm{~s}^{-1}\right)$, and Cannon et al. $\left(2002, F_{\mathrm{H} \alpha}=3.3 \times 10^{-13} \mathrm{erg} \mathrm{cm}^{-2} \mathrm{~s}^{-1}\right)$, but more than twice the Petrosian et al. (1997) value $\left(F_{\mathrm{H} \alpha}=1.9 \times\right.$ $\left.10^{-13} \mathrm{erg} \mathrm{cm}^{-2} \mathrm{~s}^{-1}\right)$. Given the general good agreement of the radio and optical measurements, we conclude not only that the spectral fit is reasonable, but also that the global optical extinction is not large enough in I Zw 18 to invalidate the comparison between the optical and radio fluxes.

The derived $E M$ of $\sim 10^{4} \mathrm{pc} \mathrm{cm}^{-6}$, combined with the source diameter of $9^{\prime \prime}$, implies that the source is optically thin; the free-free optical depth $\tau_{\mathrm{ff}}$ at $1 \mathrm{GHz}$ is 0.001 . Although the $9^{\prime \prime}(600 \mathrm{pc})$ diameter is poorly determined (see Sect. 4), we can use it to infer an electron density. We find $n_{\mathrm{e}}=4-5 \mathrm{~cm}^{-3}$, consistent with that measured density of $10 \pm 10 \mathrm{~cm}^{-3}$ in the ionized gas filaments around I Zw 18 (Izotov et al. 2001).

The non-thermal emission can be used to crudely assess the magnetic field strength in IZw 18. Assuming equipartition between the magnetic field energy and the energy of the relativistic particles, and taking the ratio of relativistic proton to electron energies to be $k=40$ (Pacholczyk 1970; Deeg et al. 1993), we calculate an equipartition $B=11 \mu \mathrm{G}$, a rather weak field. The weakness of the field depends in part on the large diameter we have assumed for the emitting region, $15^{\prime \prime}(\sim 1 \mathrm{kpc})$; if we had assumed $5^{\prime \prime}$, roughly our beam size and the lower limit on the $1.4 \mathrm{GHz}$ extent of the source (Sect. 3.1), $B$ would increase to $28 \mu \mathrm{G}$, more typical of other BCDs (Deeg et al. 1993).

\section{Radio emission in IZw 18 and star formation}

The radio emission in I Zw 18 provides another view of the star formation in the BCD and of the feedback on its ISM, complementary to the information provided by optical and ultraviolet observations. It also enables us to check the validity of the usual scaling laws for radio luminosity and star formation (Condon 1992) when applied to IZw 18.

\subsection{The windblown superbubble and feedback}

We interpret the extended radio halo and its "ears" in the context of the windblown-superbubble model described by Martin (1996). The peculiar morphology of the $1.4 \mathrm{GHz}$ and $4.8 \mathrm{GHz}$ emission could be the radio signature of a bipolar outflow, powered by supernovae ( $\mathrm{SNe}$ ) shocks and stellar winds. The HI kinematics show that the line of nodes of IZw 18 is along a position angle (PA) of $147^{\circ}$ (van Zee et al. 1998); the rotation axis would be perpendicular to this, at a $\mathrm{PA}=57^{\circ}$ in the plane of the sky. This position corresponds roughly to the "ears" emerging laterally between the NW and SE components (see Fig. 1) The calculations of Martin (1996) suggest that the polar axis of the outflow is inclined by $\sim 10^{\circ}$ to the line-of-sight, which could explain the slight asymmetry of the "ears" relative to the putative rotation axis. The radio emission is also roughly coincident with the central core of ionized gas traced by deep H $\alpha$ images (Martin 1996; Izotov et al. 2001). However, the maps (Fig. 1) and cuts (Fig. 3) suggest that the radio outflow is predominantly non-thermal, since the emission is most extended at $1.4 \mathrm{GHz}$ where non-thermal emission would tend to dominate. This would be consistent with a population of expanding $\mathrm{SNe}$ remnants creating a radio synchrotron halo. Our estimate of the non-thermal spectral index $\alpha$ is consistent with this hypothesis, since we find $\alpha \lesssim-0.5$, which is more similar to the spectral index of a $\mathrm{SN}$ remnant than that resulting from cosmic ray diffusion which occurs on a longer timescale and is characterized by a steeper spectral index $(\alpha=-0.8$, Condon 1992).

A windblown superbubble, such as the one we are invoking to explain the radio emission, should be visible at X-ray wavelengths. Indeed high-resolution Chandra observations show point source emission centered on the NW star cluster, but also diffuse emission lying between the "ears" in our radio image (Thuan et al. 2004). There is also a lack of recombination line emission in this region (Cannon et al. 2002), together with a band of extinction (Hunt et al. 2003). Thuan et al. (2004) find that the X-ray luminosity of the diffuse emission is consistent with a superbubble of radius $120 \mathrm{pc}$, expanding in an ISM with an ambient density of $n_{\mathrm{e}}=12 \mathrm{~cm}^{-3}$. The X-ray emission could be filling the cavity of a superbubble created by massive stellar winds and SNe. The radio continuum is more diffuse than the $\mathrm{X}$-ray emission, and does not appear to be directly associated with the luminous X-ray point source in the NW component. Nevertheless, the asymmetry of the radio morphology and the $\mathrm{X}$-ray emission are both suggestive of a windblown superbubble created by SNe resulting from recent massive star formation in IZw 18 (15-25 Myr ago according to Martin 1996).

Further evidence for the effect of the starburst-driven winds on the ISM includes the misalignment of the radio peaks among the different frequencies, and their misalignment with the optical continuum brightness peak of the NW component. Such misalignment of radio peaks and star clusters has been attributed to the effects of feedback (e.g., Cannon \& Skillman 2004), in which the star cluster has swept out its immediate surroundings (Johnson et al. 2003). While the thermal radio emission traces the ongoing star formation, possibly hidden by dust, the non-thermal emission probes past regions of star formation, through the synchrotron radiation of SNe cavities.

\subsection{The starburst}

The number of stars in the young clusters can be inferred from the global thermal luminosity $L_{\mathrm{T}}=1.3 \times 10^{19} \mathrm{~W} \mathrm{~Hz}^{-1}$. This corresponds to an ionizing photon flux of $1.2 \times 10^{52}$ photons $\mathrm{s}^{-1}$, or, with $10^{49}$ photons $\mathrm{s}^{-1}$ for an $\mathrm{O} 7 \mathrm{~V}$ star (Leitherer 1990), $115007 \mathrm{~V}$ equivalent ionizing stars throughout the entire main body of IZw 18 . This is in agreement with the number of O stars obtained by Hunter \& Thronson (1995). The star clusters in IZw 18 are more sparsely populated than those in the Galaxy or in nearby extragalactic systems (i.e. NGC 604 and 30 Doradus in the Large Magellanic Cloud), and are also extended in the radio, with diameters of a few hundred pc. The clusters are similar in number and size to those in NGC 625, another, higher-metallicity, BCD $\left(1 / 6 Z_{\odot}\right.$, Cannon \& Skillman 2004).

The massive SFR inferred from the thermal radio emission (Condon 1992) is $0.02 M_{\odot} \mathrm{yr}^{-1}$, and the total SFR is 
$\sim 0.1 M_{\odot} \mathrm{yr}^{-1}$, somewhat larger than the optically determined value of $0.04 M_{\odot} \mathrm{yr}^{-1}$ (Kennicutt et al. 1994). The SNe rate calculated from the thermally-derived SFR is $0.0008 \mathrm{yr}^{-1}$, which, according to the "standard" scaling relations for normal spiral galaxies (Condon 1992), would generate a non-thermal radio flux of $2.0 \mathrm{mJy}$ at $5 \mathrm{GHz}$. This is a factor of $\sim 5$ greater than of our spectral fitting result, and a factor of $\geq 2$ greater than the total observed flux. Like other BCDs, I Zw 18 does not follow the usual scaling relations for star formation and radio emission.

\subsection{Comparison with SBS 0335-052: Active vs. passive starbursts}

Despite similarly low metallicities $\left(1 / 41 Z_{\odot}\right.$, SBS 0335-052; $1 / 50 Z_{\odot}$, IZw 18), the radio properties of IZw 18 differ dramatically from those of SBS 0335-052 (Paper I). The radio emission in SBS 0335-052 is characterized by significant free-free absorption at $1.4 \mathrm{GHz}$ on a global scale, while there is no evidence for such a spectral turnover in IZw 18. Unlike the compact morphology of SBS 0335-052, the continuum in IZw 18 is extended, despite a similar global thermal/non-thermal mix (at $4.8 \mathrm{GHz}$ : 40/60 in IZw 18 and 30/70 in SBS 0335-052). The radio luminosity of IZw 18 is considerably smaller than that of SBS 0335-052; the non-thermal radio luminosity of IZw 18 is only $\sim 5 \%$, and its thermal component only about $25 \%$ of that of SBS $0335-052$. The compact X-ray source in IZw 18 is also about $25-50 \%$ as luminous as that in that in SBS 0335-052 (Thuan et al. 2004), but the total SFR in IZw 18 is more than 8 times lower. The stellar clusters are less extreme in IZw 18 than in SBS 0335-052; the superstar clusters (SSCs) in SBS 0335-052 are compact $(<25 \mathrm{pc})$ and dense $\left(\sim 100 \mathrm{pc}^{-2}\right)$ (Thuan et al. 1997; Hunt et al. 2001). The star clusters in IZw 18 are instead ordinary young clusters, $\gtrsim 300 \mathrm{pc}$ in size and $\lesssim 0.02 \mathrm{pc}^{-2}$, comparable to those in M 101 (Johnson et al. 2003), and not as extreme as in 30 Doradus (Hunter \& Thronson 1995).

Thus, in spite of their similar metal abundances, IZw 18 and SBS 0335-052 form stars in very different ways. It appears that BCDs form stars in two distinct modes, one "active" and the other one "passive" (e.g. Hirashita \& Hunt 2004). The two regimes are distinguished by the size and density of the star-forming complexes. Active star formation such as that in SBS 0335-052 occurs in dense compact regions $\left(n_{\mathrm{e}} \gtrsim\right.$ $500 \mathrm{~cm}^{-3}$, radius $\$ 100 \mathrm{pc}$ ); I Zw 18 on the other hand forms stars in a less extreme manner, with more extended and diffuse star-forming complexes. SSCs are engendered in active star formation, but not in its passive mode. Also active starbursts appear to have rising-spectrum radio sources such as those reported by Kobulnicky \& Johnson (1999) and Beck et al. $(2000,2002)$. We attribute the differences in the radio continuum properties observed in SBS 0335-052 and IZw 18 to the active/passive star formation dichotomy. While the large free-free absorption at $<1.5 \mathrm{GHz}$ in SBS 0335-052 is caused by an extremely dense compact region $\left(\sim 2000 \mathrm{~cm}^{-3}, \lesssim 20 \mathrm{pc}\right.$; Paper I), the thermal emission in I Zw 18 is apparently optically thin and diffuse.

\subsection{Comparison with the radio morphology of other BCDs}

The radio morphology of most BCDs usually shows a series of compact sources, generally thermal in nature, superimposed on a diffuse extended halo. The halo may be either thermal as in II Zw 40 (Beck et al. 2002), NGC 5253 (Beck et al. 1996), and Mrk 1039 (Yin et al. 2003), or non-thermal as in NGC 625 (Cannon \& Skillman 2004), He2-10 (Kobulnicky \& Johnson 1999), VII Zw 19 (Beck et al. 2004), and NGC 1569 (Israel \& de Bruyn 1988). As suggested by Cannon \& Skillman (2004), the radio spectral index may be used as a rough diagnostic of starburst age. Significant non-thermal radio emission, in the absence of strong non-thermal point sources such as $\mathrm{SNe}$, implies that the source is older than $\sim 10-15 \mathrm{Myr}$. This is roughly the time required for the $\mathrm{SNe}$ remnants to become detectable radio emitters, and for the onset of cosmic-ray diffusion. Thermal emission, on the other hand, whether compact or diffuse, is associated with ongoing starbursts, with timescales of $\lesssim 10 \mathrm{Myr}$, similar to that of HII regions. Optically-thick rising-spectrum bremsstrahlung sources are very young, with ages $\lesssim 1 \mathrm{Myr}$.

If we place IZw 18 in an age sequence, it would appear in the "older" group with non-thermal radio halos. On the other hand, IZw 18 and all the galaxies mentioned above (those with thermal halos: II Zw 40, NGC 5253, Mrk 1039; non-thermal halos: NGC 625, He2-10, VII Zw 19, NGC 1569) have Wolf-Rayet features in their optical spectra (Conti 1991; Guseva et al. 2000; Huang et al. 1999; Skillman et al. 2003; Schaerer et al. 1999), implying that the current starburst is no older than 4 Myr (Schaerer \& Vacca 1998). Thus in the galaxies with older non-thermal halos and the Wolf-Rayet signature of youth, there must have been a burst of star formation prior to the present one. As for those BCDs without a non-thermal radio component, either they are genuinely young, never having formed stars before the present burst, or there was a blowout creating a galactic wind which swept the cosmic-ray electrons responsible for the synchrotron emission out of the galaxy.

Prior to the present burst, IZw 18 has had a burst of star formation some $200 \mathrm{Myr}$ ago (Aloisi et al. 1999; Recchi et al. 2002; Izotov \& Thuan 2004), so it is perhaps not surprising that its diffuse radio halo is non-thermal.

\section{Conclusions}

From our study of the radio continuum at $1.4,4.8$, and $8.4 \mathrm{GHz}$ of I Zw 18, we have reached the following conclusions:

1. The radio emission in I Zw 18 has a peculiar morphology, with an extended mostly non-thermal halo, and lateral protuberances ("ears") originating from the region between the two NW and SE star clusters.

2. We interpret the extended radio halo as being consistent with a wind-blown superbubble, with a bipolar outflow oriented along the galaxy rotation axis. The non-thermal emission in the outflow may be powered by unresolved evolved $\mathrm{SNe}$ remnants. There is a possible thermal source associated with the NW star cluster.

3. By fitting a simple thermal+non-thermal model to the global fluxes, we are able to estimate the characteristics 
of the emission. IZw 18 has a 40/60 thermal/non-thermal mix at $4.8 \mathrm{GHz}$, similar to other BCDs, and optically thin thermal emission. with a thermal/total fraction of 0.30 , 0.41 , and 0.47 at $1.4,4.8$, and $8.4 \mathrm{GHz}$, respectively. Our model suggests that the emission is dominated by nonthermal emission below $\$ 8 \mathrm{GHz}$, being $\sim 70 \%$ of the total at $1.4 \mathrm{GHz}$. The fitted non-thermal spectral index $\alpha$ is $\lesssim-0.5$, flatter than the index of -0.8 found for spiral galaxies (Condon 1992). This flatter $\alpha$ is more suggestive of an ensemble of SN remnants, than of the cosmic-ray diffusion processes which operate over longer timescales. From the thermal emission, we estimate that there are $\sim 120007 \mathrm{~V}$ stars in the main body of IZw 18. The star clusters do not stand out either in number or density, resembling those in M 101. The thermal radio luminosity gives a total SFR of $0.1 M_{\odot} \mathrm{yr}^{-1}$.

4. IZw 18 does not follow the usual scaling relations for radio luminosity and SFR derived for starburst galaxies. The non-thermal radio flux predicted from using the standard relations is a factor 5 greater than the one given by spectral fit, and greater than twice the total observed flux.

5. Star formation in BCDs is bimodal. I Zw 18 appears to form stars "passively" in complexes which are diffuse and extended, while other BCDs such as SBS 0335-052 form stars "actively" in regions which are dense and compact.

Acknowledgements. We are very grateful to Doug Mink, for helping in a timely way with the astrometric calibration. While working on this paper K.K.D. was supported by a National Science Foundation Astronomy and Astrophysics Postdoctoral Fellowship ${ }^{5}$ under award AST 01-03879 and by the National Academy of Sciences through a National Research Council Fellowship at the Naval Research Laboratory. Basic research in astronomy at the Naval Research Laboratory is funded by the Office of Naval Research. T.X.T. thanks the partial support of NSF grant AST-02-05785. The authors would finally like to thank Michael Rupen for lending his considerable experience on the detection and non-detection of sources.

\section{References}

Aloisi, A., Tosi, M., \& Greggio, L. 1999, AJ, 118, 302

Beck, S. C., Turner, J. L., Ho, P. T. P., Lacy, J. H., \& Kelly, D. M. 1996, ApJ, 457, 610

Beck, S. C., Turner, J. L., \& Kovo, O. 2000, AJ, 120, 244

Beck, S. C., Turner, J. L., Langland-Shula, L. E., et al. 2002, AJ, 124, 2516

Beck, S. C., Garrington, S. T., Turner, J. L., \& Van Dyk, S. D. 2004, AJ, 128, 1552

Cannon, J. M., \& Skillman, E. D. 2004, ApJ, 610, 772

Cannon, J. M., Skillman, E. D., Garnett, D. R., \& Dufour, R. J. 2002, ApJ, 565, 931
Condon, J. J. 1992, ARA\&A, 30, 575

Conti, P. S. 1991, ApJ, 377, 115

Deeg, H., Brinks, E., Duric, N., Klein, U., \& Skillman, E. 1993, ApJ, 410,626

Gioia, I. M., Gregorini, L., \& Klein, U. 1982, A\&A, 116, 164

Guseva, N. G., Izotov, Y. I., \& Thuan, T. X. 2000, ApJ, 531, 776

Haarsma, D. B., Partridge, R. B., Windhorst, R. A., \& Richards, E. A. 2000, ApJ, 544, 641

Hirashita, H., \& Hunt, L. K. 2004, A\&A, 421, 555

Huang, J. H., Gu, Q. S., Ji, L., et al. 1999, ApJ, 513, 215

Hunt, L. K., Vanzi, L., \& Thuan, T. X. 2001, A\&A, 377, 66

Hunt, L. K., Thuan, T. X., \& Izotov, Y. I. 2003, ApJ, 588, 281

Hunt, L. K., Dyer, K. K., Thuan, T. X., \& Ulvestad, J. S. 2004, ApJ, 606, 853 (Paper I)

Hunt, L. K., Bianchi, S., \& Maiolino, R. 2005, A\&A, 434, 849

Hunter, D. A., \& Thronson, H. A., Jr. 1995, ApJ, 452, 238

Israel, F. P., \& de Bruyn, A. G. 1988, A\&A, 198, 109

Izotov, Y. I., \& Thuan, T. X. 2004, ApJ, 616, 768

Izotov, Y. I., Chaffee, F. H., Foltz, C. B., et al. 2001, ApJ, 566, 222

Johnson, K. E., Indebetouw, R., \& Pisano, D. J. 2003, AJ, 126, 101

Kennicutt, R. C., Tamblyn, P., \& Congdon, C. E. 1994, ApJ, 435, 22

Klein, U., Graeve, R., \& Wielebinski, R. 1983, A\&A, 117, 332

Klein, U., Wielebinski, R., \& Thuan, T. X. 1984, A\&A, 141, 241

Klein, U., Weiland, H., \& Brinks, E. 1991, A\&A, 246, 323

Kobulnicky, H. A., \& Johnson, K. E. 1999, ApJ, 527, 154

Kunth, D., \& Östlin, G. 2000, A\&AR, 10, 1

Leitherer, C. 1990, ApJS, 73, 1

Lequeux, J., \& Viallefond, F. 1980, A\&A, 91, 269

Loose, H.-H., \& Thuan, T. X. 1986, in Star Forming Dwarf Galaxies and Related Objects, ed. D. Kunth, T. X. Thuan, \& J. T. T. Van (Gif-sur-Yvette: Édition Frontières), 73

Martin, C. L. 1996, ApJ, 465, 680

Östlin, G. 2000, ApJ, 535, L99

Pacholczyk, A. G. 1970, Series of Books in Astronomy and Astrophysics (San Francisco: Freeman)

Papaderos, P., Izotov, Y. I., Thuan, T. X., et al. 2002, A\&A, 393, 461

Petrosian, A. R., Boulesteix, J., Comte, G., Kunth, D., \& Lecoarer, E. 1997, A\&A, 318, 390

Recchi, S., Matteucci, F., D’Ercole, A., \& Tosi, M. 2002, A\&A, 384, 799

Roussel, H., Helou, G., Beck, R., et al. 2003, ApJ, 593, 733

Sargent, W. L. W., \& Searle, L. 1970, ApJ, 162, 155

Schaerer, D., \& Vacca, W. D. 1998, ApJ, 497, 618

Schaerer, D., Contini, T., \& Pindao, M. 1999, A\&APS, 136, 35

Searle, L., \& Sargent, W. L. W. 1972, ApJ, 173, 25

Skillman, E. D., Côté, S., \& Miller, B. W. 2003, AJ, 125, 610

Thuan, T. X., Izotov, Y. I., \& Lipovetsky, V. A. 1997, ApJ, 477, 661

Thuan, T. X., Bauer, F. E., Papaderos, P., \& Izotov, Y. I. 2004, ApJ, 606, 213

van Zee, L., Westpfahl, D., Haynes, M., \& Salzer, J. 1998, AJ, 115, 1000

Yin, Q. F., Huang, J. H., \& Zheng, W. 2003, ApJ, 597, 274

Zwicky, F. 1966, ApJ, 143, 192 